\title{
Invariant Subspaces of Clustering Operators. II
}

\author{
V.A. Malyshev and R. A. Minlos
}

Department of Mathematics, Moscow State University, Moscow V-234, USSR

\begin{abstract}
Clustering operators, when restricted to $k$-particle invariant subspaces, are shown still to cluster.
\end{abstract}

\section{Introduction. The Formulation of the Main Theorem and the Plan of its Proof}

This work is the continuation of [1], where the theorem was announced that for the clustering operator with sufficiently small values of the clustering parameter (see below), a) there exist invariant " $k$-particle" subspaces, and b) the restrictions of the clustering operator upon these subspaces are unitarily equivalent to some clustering operators. In [1] part a) of this theorem was proved. Here we prove part b) constructing this unitary equivalence explicitly. For the reader's convenience this work is almost self contained.

We consider the Hilbert space $l_{2}\left(\mathbf{C}_{\mathbb{Z}^{v}}\right)$ of functions $f(T), T \in \mathbf{C}_{\mathbb{Z}^{v}}$ where $\mathbf{C}_{\mathbb{Z}^{v}}$ is the family of all finite subsets (including the empty set) of $\mathbb{Z}^{v}, v \geqq 1$. The operator $A$ in $l_{2}\left(\mathbf{C}_{\mathbb{Z}^{v}}\right)$, defined as

$$
(A f)(T)=\sum_{T^{\prime} \in \mathbf{C}_{\mathbf{Z}^{v}}} a_{T, T^{\prime}} f\left(T^{\prime}\right) \quad T \in \mathbf{C}_{\mathbb{Z}^{v}}
$$

and commuting with unitary group $\left\{U_{t}, t \in \mathbb{Z}^{\nu}\right\}$ of translations in $l_{2}\left(\mathbf{C}_{\mathbb{Z}^{v}}\right)$ :

$$
\left(U_{t} f\right)(T)=f(T-t), \quad T \in \mathbf{C}_{\mathbb{Z}^{v}}, \quad t \in \mathbb{Z}^{v},
$$

$T-t=\left\{x_{i}-t, i=1,2 \ldots,|T|\right\}$, if $T=\left\{x_{i}, i=1,2, \ldots|T|\right\}$ and $x_{i} \in \mathbb{Z}^{v}, i=1,2 \ldots,|T|$, is called clustering if its matrix elements $a_{T, T^{\prime}}$ satisfy the cluster expansion

$$
a_{T, T^{\prime}}=\sum_{k \geqq 1} \sum_{\left\{\tau_{1}, \ldots, \tau_{k}\right\}} \omega_{k}\left(\tau_{1}, \ldots, \tau_{k}\right)
$$

Let $Y_{0}=\{0\} \times \mathbb{Z}^{v} \subset \mathbb{Z}^{v+1}$ be a zero-time slice of $\mathbb{Z}^{v+1}$ and $Y_{1}=\{1\}$ $\times \mathbb{Z}^{v} \subset \mathbb{Z}^{v+1}$. Let $\pi_{0}: \mathbb{Z}^{v} \rightarrow Y_{0}$ and $\pi_{1}: \mathbb{Z}^{v} \rightarrow Y_{1}$ be identity maps. We define $\tau_{i}$ to be a pair $\left(T_{i}, T_{i}^{\prime}\right)$ of subsets of $\mathbb{Z}^{\nu}$. We shall often identify $\tau_{i}$ with the subset $\pi_{0}\left(T_{i}\right) \cup \pi_{1}\left(T_{i}^{\prime}\right)$ of $Y_{0} \cup Y_{1}$. The summation in (1.3) is over all partitions $\left(\tau_{1}, \ldots, \tau_{k}\right)$ of $\pi_{0}(T) \cup \pi_{1}\left(T^{\prime}\right) \equiv\left(T, T^{\prime}\right)$. 
It is assumed that $\omega_{k}$ are symmetric functions satisfying the following conditions:

0)

$$
\omega_{1}(\emptyset, \emptyset)=0
$$

and if either $T_{i}=\emptyset$ or $T_{i}=\emptyset$ for some $i$ then

$$
\omega_{k}\left(\tau_{1}, \ldots, \tau_{k}\right)=0, \quad k=1,2, \ldots
$$

1) For any $\tau_{1}, \ldots, \tau_{k}$ and any $s_{1}, s_{2}, \ldots, s_{k} \in \mathbb{Z}^{v}$

$$
\omega_{k}\left(\tau_{1}+s_{1}, \ldots, \tau_{k}+s_{k}\right)=\omega_{k}\left(\tau_{1}, \ldots, \tau_{k}\right) .
$$

where for $\tau=\left(T, T^{\prime}\right)$

$$
\tau+s=\left(T+s, T^{\prime}+s\right), \quad s \in \mathbb{Z}^{v} .
$$

If for some $i, j,\left(\tau_{i}+s_{i}\right) \cap\left(\tau_{j}+s_{j}\right) \neq \emptyset$ then (1.6) is the unambiguous definition of $\omega_{k}$ in such cases. So $\omega_{k}\left(\tau_{1}, \ldots, \tau_{k}\right)$ if defined for all $\tau_{1}, \ldots, \tau_{k}$

2) There exists $\beta, 0<\beta<1$ (clustering parameter), $M>0$ and translation invariant integer-valued metrics $\varrho$ on $\mathbb{Z}^{v+1}$ such that for any $k, \tau_{i}, \ldots, \tau_{k}$,

$$
\left|\omega_{k}\left(\tau_{1}, \ldots, \tau_{k}\right)\right| \leqq M \prod_{i=1}^{k} \beta^{d_{t_{i}}},
$$

where $d_{\tau}$ is the minimum length of the tree connecting points of $\tau \subset Y_{0} \cup Y_{1}$ and the lengths of edges of this tree are measured in the metrics $\varrho$.

It was proved in [3] that the transfer-matrix of the Ising model for hightemperatures $\beta^{-1}$ is the clustering operator if we take

$$
\varrho\left(t_{1}, t_{2}\right)=\sum_{i=1}^{v+1}\left|t_{1}^{(i)}-t_{2}^{(i)}\right|, \quad t_{j}=\left(t_{j}^{(1)}, \ldots, t_{j}^{(v+1)}\right), \quad j=1,2 .
$$

We shall consider mainly the metrics (1.8) for the sake of simplicity.

Let us define subspaces: $n=0,1,2, \ldots$,

$$
\begin{aligned}
& L^{n}=\{f: f(T)=0, \quad|T| \neq n\} \subset l_{2}\left(\mathbf{C}_{\mathbb{Z}^{n}}\right) \\
& L^{V}=\bigoplus_{n \in V} L^{n}, \quad V \subset\{0,1, \ldots\} \equiv[0, \infty) .
\end{aligned}
$$

Operators $A$ acting in $L^{V}$ will often be considered to be defined in all $l_{2}\left(\mathbf{C}_{\mathbb{Z}^{v}}\right)$ in the following way

$$
A f=0 \text { for } f \in L^{[0, \infty) \backslash V} .
$$

Let the selfadjoint clustering operator $A$ be given; we define the selfadjoint operator $B$ with matrix elements

$$
b_{T, T^{\prime}}=\left\{\begin{array}{l}
0,|T| \neq\left|T^{\prime}\right| \\
\sum \omega_{k}\left(\left(\left\{t_{1}\right\},\left\{t_{i_{1}}^{\prime}\right\}\right), \ldots,\left(\left\{t_{k}\right\},\left\{t_{i_{k}}^{\prime}\right\}\right)\right) \\
\text { if } T=\left\{t_{1}, \ldots, t_{k}\right\} \subset Y_{0}, T^{\prime}=\left\{t_{1}^{\prime}, \ldots, t_{k}^{\prime}\right\} \subset Y_{i},
\end{array}\right.
$$


and summation is over all permutations $\left(\begin{array}{c}1 \ldots k \\ i_{1} \ldots i_{k}\end{array}\right)$ such that $\varrho\left(t_{s}, t_{i_{s}}^{\prime}\right)=1$ for $s=1, \ldots, k$. Then $B$ is called the main part (main symbol) of $A$. The operator $A$ is called regular if there exist constants $C>0$ and $L>0$ such that

$$
B \geqq B_{0} \text {, }
$$

where $B_{0}=B_{0}(C, L)$ is diagonal and its matrix elements

$$
b_{T, T^{\prime}}=L(C \beta)^{|T|} \delta_{T, T^{\prime}}
$$

Remark. In the metrics (1.8) $B$ itself is diagonal and its matrix elements are equal to

$$
b_{T, T^{\prime}}=\delta_{T, T^{\prime}} \omega_{k}\left(\left(\left\{t_{1}\right\},\left\{t_{1}\right\}\right), \ldots,\left(\left\{t_{k}\right\},\left\{t_{k}\right\}\right)\right) .
$$

So here regularity means that

$$
\omega_{k}\left(\left(\left\{t_{1}\right\},\left\{t_{1}\right\}\right), \ldots,\left(\left\{t_{k}\right\},\left\{t_{k}\right\}\right)\right)>L(C \beta)^{k} .
$$

The main result of [1] is

Theorem 1.1. Let $\mathfrak{A}=\mathfrak{U}(M, \beta, \varrho, L, C)$ be the class of selfadjoint regular clustering operators satisfying (1.7) and (1.11). Then for any integer $N \geqq 1$ there exists $\beta_{0}=\beta_{0}(N, M, L, \varrho, C)$, such that for all $\beta<\beta_{0}$ any operator $A \in \mathfrak{U}$ has $N+2$ mutually orthogonal invariant subspaces $\mathscr{H}_{0}=L^{0}, \mathscr{H}_{1}, \ldots, \mathscr{H}_{N}, \overline{\mathscr{H}}_{N} \subset l_{2}\left(\mathbf{C}_{\mathbb{Z}^{v}}\right)$. They are invariant also with respect to translations $\left\{U_{t}, t \in \mathbb{Z}^{v}\right)$. The spectrum $\sigma\left(\left.A\right|_{\mathscr{H}_{k}}\right)$ of the restriction of $A$ onto $\mathscr{H}_{k}$ satisfies the following conditions

$$
\begin{aligned}
& \sigma\left(\left.A\right|_{\mathscr{H}_{k}}\right) \subseteq\left[K_{2} \beta^{k}, \quad K_{1} \beta^{k}\right], \quad k=1,2, \ldots, N, \\
& \sigma\left(\left.A\right|_{\mathscr{H}_{N}}\right) \subseteq\left[-K_{1} \beta^{N+1}, \quad K_{1} \beta^{N+1}\right],
\end{aligned}
$$

where $K_{1}>K_{2}>0, K_{i}=K_{i}(N, L, M, C, \varrho) i=1,2$ do not depend on $\beta$. Of course $\sigma\left(\left.A\right|_{\mathscr{H}_{0}}\right)=\{0\}$.

Here we prove the following.

Theorem 1.2. Assuming conditions of Theorem 1.1, let $A^{(k)}, U_{t}^{(k)}$ be restrictions of $A$ and $U_{t}$ onto $\mathscr{H}_{k}, k=1, \ldots, N$. Then there exist unitaries:

$$
V_{k}: \mathscr{H}_{k} \rightarrow L^{k}, \quad k=1, \ldots, N,
$$

such that

$$
V_{k} U_{t}^{(k)} V_{k}^{-1}=\left.U_{t}\right|_{L^{k}}
$$

and moreover $V_{k} A^{(k)} V_{k}^{-1}$ in $L^{k}$ is clustering.

Let us recall the construction of invariant subspaces $\mathscr{H}_{k}$. For this, first the increasing sequence of invariant subspaces

$$
\mathscr{H}_{0}=\mathscr{L}_{0} \subset \mathscr{L}_{1} \subset \mathscr{L}_{2} \subset \ldots \subset \mathscr{L}_{N} \subset l_{2}\left(\mathbf{C}_{\mathbb{Z}^{v}}\right)
$$


is constructed and then we put

$$
\begin{aligned}
& \mathscr{H}_{1}=\mathscr{L}_{1} \ominus \mathscr{L}_{0}, \\
& \mathscr{H}_{2}=\mathscr{L}_{2} \ominus \mathscr{L}_{1}, \\
& \ldots . . \\
& \mathscr{H}_{N}=\mathscr{L}_{N} \ominus \mathscr{L}_{N-1}, \\
& \overline{\mathscr{H}}_{N}=l_{2}\left(\mathbf{C}_{\mathbb{Z}^{v}}\right) \ominus \mathscr{L}_{N} .
\end{aligned}
$$

To construct (1.17) we put, for $k>0$

$$
L \leqq k=L^{[0, k]} \text { and } L^{>k}=L^{[L-k+1, \infty)} .
$$

Then

$$
l_{2}\left(\mathbf{C}_{\mathbb{Z}^{v}}\right)=L^{\leqq k} \oplus L^{>k}
$$

and $A$ has a block structure

$$
A=\left\|\begin{array}{c}
A_{11}^{(k)}, A_{12}^{(k)} \\
A_{21}^{(k)}, A_{22}^{(k)}
\end{array}\right\|,
$$

where $A_{11}^{(k)}=P_{L^{\leqq k}} A P_{L^{\leqq k}}$ and so on. Here $P_{Q}$ is the orthogonal projection onto $Q \subset l_{2}\left(\mathbf{C}_{\mathbb{Z}^{*}}\right)$. We shall look for $\mathscr{L}_{k, 1} \mid \leqq k \leqq N$ as a graph

$$
\mathscr{L}_{k}=\left\{\varphi+S^{(k)}, \quad \in L^{\leqq k}\right\}
$$

of some mapping $S^{(k)}$

(We recall that we consider $S^{(k)}$ defined on $l_{2}\left(\mathbf{C}_{\mathbb{Z}^{*}}\right)$, and we define it to be 0 on $L \geqq k$.) Invariance of $\mathscr{L}_{k}$ with respect to $A$ is equivalent then to the following equation with respect to

$$
S^{(k)}=A_{12}^{(k)}\left(A_{11}^{(k)}\right)^{-1}+A_{22}^{(k)} S^{(k)}\left(A_{11}^{(k)}\right)^{-1}-S^{(k)} A_{12}^{(k)} S^{(k)}\left(A_{11}^{(k)}\right)^{-1} .
$$

It was proved in [1] that for small $\beta(1.21)$ has the unique solution $S^{(k)}$ with $\left\|S^{(k)}\right\|$ sufficiently small. In Sect. 3 we prove

Lemma 1.3. For sufficiently small $\beta$, i.e. for $\beta<\beta_{0}=\beta_{0}(N, L, M, \varrho, C)$, all $S^{(k)}$, $k=1, \ldots, N$, are the clustering operators with clustering parameter $\beta^{\lambda}, \lambda>0$.

Let us sketch the proof of the main theorem. One can find details in the next sections. We must find in $\mathscr{H}_{k}, k=1,2, \ldots, N$, the orthonormal basis

$$
\left\{h_{T}^{(k)}, \quad T \in \mathbf{C}_{\mathbb{Z}^{v}}, \quad|T|=k\right\},
$$

such that for each $t \in \mathbb{Z}^{v}$ and $T$,

$$
U_{t}^{(k)} h_{T}^{(k)}=h_{T+t}^{(k)},
$$

and matrix elements of $A^{(k)}$ in this basis

$$
\left(A^{(k)} h_{T}^{(k)}, h_{T^{\prime}}^{(k)}\right) \equiv \tilde{a}_{T, T^{\prime}}^{(k)}
$$

admit the cluster expansion with some clustering parameter

$$
\widetilde{\beta}=\widetilde{\beta}(\beta), \quad \tilde{\beta} \rightarrow 0 \quad \text { if } \quad \beta \rightarrow 0 .
$$


Whe shall look for $\left\{h_{T}^{(k)}\right\}$ as

$$
h_{T}^{(k)}=V^{(k)} e_{T}
$$

where $e_{T}\left(T^{\prime}\right)=\delta_{T, T^{\prime}}$ and operator $V^{(k)}$ is such that:

1) $\operatorname{Ker} V^{(k)}=l_{2}\left(C_{\mathbb{Z}^{v}}\right) \ominus L^{k}, \operatorname{Im} V^{(k)}=\mathscr{H}_{k}$,

2) $V^{(k)}: L^{k} \rightarrow \mathscr{H}_{k}$ is unitary,

3) $V^{(k)}=P_{L^{k}}+\Gamma^{(k)}$,

where $\Gamma^{(k)}$ is clustering with its norm sufficiently small. It follows from (1.23) and (1.24) that

$$
\tilde{a}_{T, T^{\prime}}^{(k)}=\left(\left(V^{(k)}\right)^{*} A V^{(k)}\right)_{T, T^{\prime}}=a_{T, T^{\prime}}+\left(\left(\Gamma^{(k)}\right)^{*} A\right)_{T, T^{\prime}}+\left(A \Gamma^{(k)}\right)_{T, T^{\prime}}+\left(\left(\Gamma^{(k)}\right)^{*} A \Gamma^{(k)}\right)_{T, T^{\prime}}
$$

Here $(B)_{T, T^{\prime}}$ are the matrix elements of $B$ in the basis $\left\{e_{T}, T \in \mathbf{C}_{\mathbb{Z}^{v}}\right\}$. We prove in the following sections that the product of clustering operators is the clustering operator again, and so $\left(\Gamma^{(k)}\right)^{*} A, A \Gamma^{(k)},\left(\Gamma^{(k)}\right)^{*} A \Gamma^{(k)}$ are clustering.

From this and from (1.25) it follows that matrix elements $\tilde{a}_{T, T^{\prime}}^{(k)}$ obey the cluster expansion. The equality (1.16) follows from (1.22). So we must construct $V^{(k)}$ with all these properties. First we construct $\tilde{V}^{(k)}$ with all these properties except unitarity.

Lemma 1.4. The operator

$$
\tilde{V}^{(k)}=\left(E-P_{\mathscr{L}_{k-1}}\right)\left(E+S^{(k)}\right) P_{L^{(k)}}
$$

has the properties 1) and 3) of $V^{(k)}$.

See the proof in Sect. 3.

Let us consider the system

$$
\left\{\tilde{h}_{T}^{(k)}=\tilde{V}^{(k)} e_{T}, \quad|T|=k\right\} .
$$

This system is complete in $\mathscr{H}_{k}$ and its fundamental matrix is equal to

$$
C_{T, T^{\prime}}^{(k)}=\left(\tilde{h}_{T}^{(k)}, \tilde{h}_{T^{\prime}}^{(k)}\right)=\left(\left(\tilde{V}^{(k)}\right)^{*} \tilde{V}^{(k)}\right)_{T, T^{\prime}}, \quad|T|=\left|T^{\prime}\right|=k .
$$

Let us note that

$$
D^{(k)}=\left(\tilde{V}^{(k)}\right)^{*} \tilde{V}^{(k)}
$$

is selfadjoint positive and $\operatorname{Ker} D^{(k)}=l_{2}\left(\mathbf{C}_{\mathbb{Z}^{v}}\right) \ominus L^{k}$. $\operatorname{Im} D^{(k)}=L^{k}$.

Lemma 1.5. The operator $\left(D^{(k)}\right)^{-1 / 2}$ is defined in $L^{k}$ and

$$
\left(D^{(k)}\right)^{-1 / 2}=P_{L^{k}}+\Delta^{(k)},
$$

where $\Delta^{(k)}$ is selfadjoint clustering operator in $L^{k}$ [i.e. $\operatorname{Im} \Delta^{(k)}=L^{k}, \operatorname{Ker} \Delta^{(k)}$ $\left.=l_{2}\left(\mathbf{C}_{\mathbb{Z}^{v}}\right) \ominus L^{k}\right]$. See the proof in Sect. 3 .

It is evident that $V^{(k)}=\left.\tilde{V}^{(k)}\left(D^{(k)}\right)^{-1}\right|^{2}$ maps unitarily $L^{k}$ onto $\mathscr{H}_{k}$. Moreover $V^{(k)}=\left(P_{L^{k}}+\tilde{\Gamma}^{(k)}\right)\left(P_{L^{k}}+\Delta^{(k)}\right)=P_{L^{k}}+\tilde{\Gamma}^{(k)} P_{L^{k}}+P_{L^{k}} \Delta^{(k)}+\tilde{\Gamma}^{(k)} \Delta^{(k)}=P_{L^{k}}+\Gamma^{(k)}$,

where

$$
\Gamma^{(k)}=\Gamma^{(k)} P_{L^{k}}+P_{L^{k}} \Delta^{(k)}+\tilde{\Gamma}^{(k)} \Delta^{(k)} .
$$

Now the following Lemma 1.6 completes the proof of the Theorem 1.2. 
Lemma 1.6. The operator $\Gamma^{(k)}$ is clustering.

See the proof in Sect. 3 .

\section{The Algebra of Clustering Operators}

Let $A_{0}, \ldots, A_{k-1}$ be clustering operators and let their cluster functions $\omega_{s}^{A_{i}}\left(\tau_{1}, \ldots, \tau_{s}\right), i=0, \ldots, k-1$ satisfy the following bounds

$$
\left|\omega_{s}^{A_{i}}\left(\tau_{1}, \ldots, \tau_{s}\right)\right| \leqq M_{i} \prod_{j=1}^{s} \beta^{d_{\tau_{j}}} .
$$

Let us put

$$
Y_{i}=\{i\} \times \mathbb{Z}^{v} \subset \mathbb{Z}^{v+1} T^{i}=\pi_{i} T, \quad T \subset \mathbb{Z}^{v},
$$

and $\pi_{i}$ is the identity map $\pi_{i}: \mathbb{Z}^{v} \rightarrow Y_{i}$.

Theorem 2.1. Let (2.1) hold. Then the operator $B=A_{k-1} \ldots A_{1} A_{0}$ is clustering and its cluster functions

$$
\left|\omega_{s}^{B}\left(\tau_{1}, \ldots, \tau_{s}\right)\right| \leqq \prod_{i=0}^{k-1} M_{i} \prod(C \beta)^{d} T_{j}^{0} \cup \tilde{T}_{j}^{k}, \tau_{j}=\left(T_{j}, \tilde{T}_{j}\right)
$$

where $C$ is some absolute constant (not depending on $s, \beta, k, A_{0}, \ldots A_{k-i}$, but depending on v).

Proof. Let $\gamma=\left\{\gamma_{j}^{i}\right\}$ be any finite (unordered) system of finite subsets of $Y=\bigcup_{0}^{k} Y_{i} \subset \mathbb{Z}^{v+1}$ such that
a) $\gamma_{j}^{i} \subset Y_{i} \cup Y_{i+1}, \quad \gamma_{j}^{i} \cap Y_{i} \neq \emptyset, \gamma_{j}^{i} \cap Y_{i+1} \neq \emptyset$, for any $i=0,1, \ldots, k-1$,
b) $\left(\bigcup_{j} \gamma_{j}^{i-1}\right) \cap Y_{i}=\left(\bigcup_{j} \gamma_{j}^{i}\right) \cap Y_{i}$
c) $\gamma_{j}^{i} \cap \gamma_{j^{\prime}}^{i}=\emptyset$ for any $j \neq j^{\prime}$ and any $i$,
d) $\gamma$ is connected (see $[3$, p. 9]).

Let us call a bond any such system $\gamma$ and put

$$
\tilde{\gamma}=\bigcup_{i, j} \gamma_{j}^{i}, \quad T^{i}(\gamma)=\tilde{\gamma} \cap Y_{i}
$$

We shall consider also finite (unordered) systems $\Gamma=\{\gamma(1), \ldots, \gamma(m)\}$ of bonds. Let us call such system $\Gamma$ regular if

$$
\begin{array}{ll}
T^{0}(\gamma(i)) \cap T^{0}(\gamma(j))=\emptyset, & i \neq j \\
T^{k}(\gamma(i)) \cap T^{k}(\gamma(j))=\emptyset, & i \neq j
\end{array}
$$

and completely regular if

$$
\tilde{\gamma}(i) \cap \tilde{\gamma}(j)=\emptyset, \quad i \neq j .
$$


Let us denote for any $\Gamma$

$$
\tilde{\Gamma}=\bigcup_{i=1}^{m} \gamma(i)
$$

So $\tilde{\Gamma}$ is the system of subsets $\left\{\gamma_{j}^{i}(k)\right\}, \Gamma$ is called connected if $\tilde{\Gamma}$ is connected $([2,3])$.

Let $\Gamma$ be given and let $\tau^{i}(\Gamma)$ be the system of all $\gamma_{j}^{i}(k)$ for all possible $j$ and $k$. Let $s_{i}$ be their number and $\tau_{1}, \ldots, \tau_{s_{i}} \subset Y_{0} \cup Y_{1}$ be their translations by the vector $(-i, 0, \ldots, 0)$. Then we put

$$
\begin{aligned}
\omega^{A_{i}}\left(\tau^{i}(\Gamma)\right) & =\omega_{s_{i}}^{A_{i}}\left(\tau_{1}, \ldots, \tau_{s_{i}}\right) \\
\omega(\Gamma) & =\omega_{s_{0}}^{A_{0}}\left(\tau^{0}(\Gamma)\right) \cdot \omega_{s_{1}}^{A_{1}}\left(\tau^{1}(\Gamma)\right) \ldots \omega_{s_{k-1}}^{A_{k-1}}\left(\tau^{k-1}(\Gamma)\right) .
\end{aligned}
$$

If $b_{T^{0}, T^{k}}$ are matrix elements of $B$ then

$$
b_{T^{0}, T^{k}}=\sum_{T^{1}, T^{2}, \ldots, T^{k-1}} a_{T^{0}, T^{1}}^{A_{0}} a_{T^{1}, T^{2}}^{A_{1}} \ldots a_{T^{k-1}, T^{k}}^{A_{k-1}}
$$

One can see from (2.9) and (2.10) that

$$
b_{T^{0} T^{k}}=\sum_{\Gamma} \omega(\Gamma)
$$

where the summation is over all completely regular $\Gamma=\{\gamma(1), \ldots, \gamma(m)\}$ such that

$$
T^{0}(\Gamma) \equiv \bigcup_{j=1}^{m} T^{0}(\gamma(j))=T^{0}, \quad T^{k}(\Gamma) \equiv \bigcup_{j=1}^{m} T^{0}(\gamma(j))=T^{k}
$$

Definition.

$$
\omega_{s}^{B}\left(\left(T_{1}, \tilde{T}_{1}\right), \ldots\left(T_{s}, \tilde{T}_{s}\right)\right) \stackrel{\text { def }}{=} \sum D\left(\Gamma_{1}\right) \ldots D\left(\Gamma_{s}\right) \omega\left(\Gamma_{1} \cup \Gamma_{2} \cup \ldots \cup \Gamma_{s}\right),
$$

where the summation is over all regular $\Gamma_{1}, \ldots, \Gamma_{s}$ such that the system $\tilde{\Gamma}_{l}$, $l=1, \ldots, s$ is connected and

$$
T^{0}\left(\Gamma_{l}\right)=T_{l}^{0}, \quad T^{k}\left(\Gamma_{l}\right)=\tilde{T}_{l}^{k}, \quad l=1, \ldots, s .
$$

To define $D(\Gamma)$ for any $\Gamma=\{\gamma(1), \ldots, \gamma(m)\}$, let us consider graph $G=G_{\Gamma}$ with vertices $1, \ldots, m$. There is the (inique) line between vertices $i$ and $j$ iff $\tilde{\gamma}(i) \cap \tilde{\gamma}(j) \neq \emptyset$. Then we put

$$
D(\Gamma)=\mu_{\mathfrak{2}_{G}}(\mathbb{\Phi}, \mathbb{I})
$$

(see [2,3]), i.e. $D(\Gamma)$ is the Möbius function for the lattice $\mathfrak{A}_{G}$ (see $[2,3]$ )

Lemma 2.2 If $\omega_{s}^{B}$ are defined by (2.13) then

$$
b_{T, \tilde{T}}=\sum \omega_{s}^{B}\left(\left(T_{1}, T_{1}\right), \ldots\left(T_{s}, T_{s}\right)\right),
$$

where the summation is over all $s, T_{i}, T_{i}$ such that

$$
T=\cup T_{i}, \quad \widetilde{T}=\cup \tilde{T}_{i} \quad T_{i} \cap T_{j}=\emptyset, \quad \tilde{T}_{i} \cap \tilde{T}_{j}=\emptyset, \quad i \neq j .
$$

To prove this lemma it is sufficient to consider the sum

$$
\sum D\left(\Gamma_{1}\right) \ldots D\left(\Gamma_{s}\right)
$$


over all $\Gamma_{1}, \ldots, \Gamma_{s}$ such that $\Gamma_{1} \cup \Gamma_{2} \cup \ldots \cup \Gamma_{s}=\Gamma$ for some fixed $\Gamma$ and to prove that this sum is equal to 1 for completely regular $\Gamma$ and zero otherwise. But this easily follows from the definition of the Möbius function and formula (1.14), Chapter II of [3] (see also [2]).

Lemma 2.3. $\omega_{s}^{B}$ are translation invariant, i.e. (1.6) holds for them.

This is evident from the definition (2.13) as the summation in (2.13) is over independent $\Gamma_{1}, \ldots, \Gamma_{s}$.

Remark 2.1. Obtaining translation invariance was the main reason for the definition of $\omega_{s}^{B}$. One can begin with the sum over all completely regular $\Gamma_{1}, \ldots, \Gamma_{s}$ with $D\left(\Gamma_{i}\right) \equiv 1$ and then compensate nonregularity of $\Gamma_{1} \cup \Gamma_{2} \cup \ldots \cup \Gamma_{s}$ by induction. We note also that if $B$ is clustering then its clustering function $\omega_{s}^{B}$ are uniquely defined.

To end the proof of Theorem 2.1 we shall prove (2.2). We use Lemma II.1.2 and Theorem II.2.2 of [3] (see also [2]) to eliminate $D(\Gamma)$. More exactly if $\Gamma=\{\gamma(1), \ldots, \gamma(m)\}$, then

$$
|D(\Gamma)| \leqq \prod_{k=1}^{m} C^{d_{\tilde{\gamma}^{\prime}(k)}} \leqq \prod_{i, j, k} C^{d_{\gamma_{j}^{\prime}(k)}}
$$

and $C=C(v)$ is an absolute constant.

So

$$
\left|\omega_{s}^{B}\left(\tau_{1}, \ldots,, \tau_{s}\right)\right| \leqq \prod_{i=0}^{k-1} M_{A_{i}} \sum_{\Gamma_{1}, \ldots, \Gamma_{s}} \prod_{i, j, k, l}(C \beta)^{d_{j}(k, l)}
$$

where

$$
\Gamma_{l}=\{\gamma(k, l)\}, \quad l=1, \ldots, s \quad \tau_{i}=\left(T_{i}, \widetilde{T}_{i}\right) \quad i=1, \ldots, s .
$$

Let us note that for any $\Gamma_{1}, \ldots, \Gamma_{s}$

$$
\sum_{i, j, k . l} d_{v_{j}^{i}(k, l)} \geqq \sum_{i=1}^{s} d_{T_{i}^{0} \cup \tilde{T}_{i}^{k}}
$$

To prove (2.2) let us consider arbitrary sequences $\left(\delta_{1}, \ldots, \delta_{N}\right)$ of subsets of $Y=\bigcup_{0}^{k} Y_{i}$ containing point $0 \in \mathbb{Z}^{v+1},\left|\delta_{i}\right| \geqq 2$. It is clear that

$$
\sum_{N} \sum_{d_{\delta_{1}}+\cdots+d_{\delta_{N}} \geqq d} 3^{N}(C \beta)^{d_{\delta_{1}}+d_{\delta_{2}}+\cdots+d_{\delta_{N}}} \leqq\left(C_{1} \beta\right)^{d}
$$

for some absolute constant $C_{1}$.

Now we shall describe the mapping of the set of such sequences $\left(\delta_{1}, \ldots, \delta_{N}\right)$ onto the set of $\left\{\Gamma_{1}, \ldots, \Gamma_{s}\right\}$. As the first step we choose the first (in lexicographic order) point $t_{0}$ of $T_{0}$ and translate $\delta_{1}$ to thos point. As the $k^{\text {th }}$ step we must decide whether to translate $\delta_{k}$ to the next point (in lexicographic order) of the set $T^{0} \cup\left\{\bigcup_{i=1}^{k-1} \widetilde{\delta}_{i}\right\}, \widetilde{\delta}_{i}$ is the translation of $\delta_{i}$ constructed in earlier steps, or to translate $\delta_{k}$ to the point of $(k-1)$ step, or to return to $T^{0}$ and begin construction from the very beginning, thus constructing new $\gamma(k, l)$. It is evident that any $\left\{\Gamma_{1}, \ldots, \Gamma_{s}\right\}$ can be constructed in this way. As we have 3 possibilities on each step, Theorem 2.1 follows from (2.20). 
Remark 2.2. If $F$ is transfer-matrix then the cluster property of $F^{k}$ for any $k$ follows also from the results of [3] if instead of $Y_{0} \cup Y_{1}$ one considers $Y_{0} \cup Y_{k}$.

\section{Corollary 2.4.}

$$
\left|\omega_{s}^{B}\left(\tau_{1}, \ldots, \tau_{s}\right)\right| \leqq \prod_{i=1}^{s} M_{i}(C \beta)^{(k-1) s} \prod_{i=1}^{s}(C \beta)^{d_{\tau_{i}}}
$$

This follows from

$$
d_{T^{0} \cup \tilde{T}^{k}} \geqq d_{\tau}+k-1
$$

for any

$$
\tau=(T, \tilde{T})
$$

\section{The End of Proof of Theorem 1.2}

Lemma 3.1. For $\beta$ sufficiently small, $\beta<\beta_{0}(N)$, the operator $\left(A_{11}^{(k)}\right)^{-1}=G_{k}$ in $L \leqq k$ is clustering and its cluster functions satisfy the following estimate

$$
\left|\omega_{s}^{G_{k}}\left(\tau_{1}, \ldots, \tau_{s}\right)\right|<C_{N}\left(\beta^{-1}\right)^{N(1+\lambda)} \prod_{1}^{s}\left(\beta^{\lambda}\right)^{d_{\tau_{i}}}
$$

for any $\lambda, 0<\lambda<\frac{1}{N+1}$ and $C_{N}$ is the absolute constant.

Proof. One has

$$
A_{11}^{(k)}=B_{11}^{(k)}+\tilde{A}_{11}^{(k)}
$$

where $B_{11}^{(k)}$ is the main symbol of $A_{21}^{(k)}, \tilde{A}_{11}^{(k)}=A_{11}^{(k)}-B_{11}^{(k)}, B_{11}^{(k)}$ is diagonal if the metrics is (1.8). We consider this matrics for simplicity. Then $\left(B_{11}^{(k)}\right)^{-1}$ is also diagonal and its matrix elements satisfy the bound

$$
\left|\left(B_{11}^{(k)}\right)_{T, T}^{-1}\right|<\left(C_{0} \beta\right)^{-2|T|},|T| \leqq k .
$$

We have

$$
\left(A_{11}^{(k)}\right)^{-1}=\left(B_{11}^{(k)}\right)^{-1 / 2}\left(E+\left(B_{11}^{(k)}\right)^{-1 / 2} \tilde{A}_{11}^{(k)}\left(B_{11}^{(k)}\right)^{-1 / 2}\right)^{-1}\left(B_{11}^{(k)}\right)^{-1 / 2} .
$$

Let us put

$$
V^{(k)}=\left(B_{11}^{(k)}\right)^{-1 / 2} \tilde{A}_{11}^{(k)}\left(B_{11}^{(k)}\right)^{-1 / 2} .
$$

Then $V^{(k)}$ is clustering and its cluster functions

$$
\left|\omega_{s}^{\zeta^{k(k)}}\left(\tau_{1}, \ldots, \tau_{s}\right)\right| \leqq\left(C_{0} \beta\right)^{-\frac{1}{2} \sum_{1}^{s}\left|\tau_{i}\right|} \prod^{s} \beta^{d_{\tau_{i}}}
$$

The main symbol of $V^{(k)}$ is zero. So $\omega_{s}^{V^{(k)}}\left(\tau_{1}, \ldots, \tau_{s}\right)$ do not equal 0 only for those $\tau_{1}, \ldots, \tau_{s}$ where $d_{\tau_{i}} \geqq 2$ for at least one $i$. So

$$
\sum d_{\tau_{i}} \geqq \frac{1}{2} \sum_{i=1}^{s}\left|\tau_{i}\right|+1
$$


Then for any $\lambda^{\prime}, 0<\lambda^{\prime}<1$

$$
\left(C_{0} \beta\right)^{-\frac{1}{2} \sum\left|\tau_{i}\right|} \cdot \beta^{\sum d_{\tau_{i}}} \leqq C_{0}^{-\frac{1}{2} \sum \tau_{i}}(\beta)^{1-\lambda^{\prime}-\frac{\lambda s}{2} \sum_{1}^{s}\left|\tau_{i}\right|} \cdot \prod\left(\beta^{\lambda^{\prime}}\right)^{d \tau_{i}}
$$

Using

$$
\sum_{1}^{s}\left|\tau_{i}\right| \leqq 2 N
$$

one can find for any $\lambda^{\prime}<(N+1)^{-1}$ that

$$
\left|\omega_{s}^{\bigvee^{(k)}}\left(\tau_{1}, \ldots, \tau_{s}\right)\right| \leqq C_{0}^{-N} \prod_{1}^{s}\left(\beta^{\lambda^{\prime}}\right)^{d_{\tau_{i}}}
$$

As the norm of $V^{(k)}$ does not exceed 1 for $\beta$ sufficiently small, we have

$$
\left(E+V^{(k)}\right)^{-1}=E-V^{(k)}+\sum_{m=2}^{\infty}\left(V^{(k)}\right)^{m}(-1)^{m} .
$$

Using Theorem 2.1 and Corollary 2.4 we get that the operator $\left(V^{(k)}\right)^{m}$ is clustering and its cluster functions satisfy the following estimates $(m>i)$

$$
\left|\omega_{s}^{\left(V^{(k) m}\right.}\left(\tau_{1}, \ldots, \tau_{s}\right)\right| \leqq C_{0}^{-N m}\left(C \beta^{\lambda}\right)^{(m-1) s} \prod_{1}^{s}\left(C \beta^{\lambda}\right)^{d_{\tau_{i}}}
$$
It follows that the operator $\sum_{2}^{\infty}\left(V^{(k)}\right)^{m}(-1)^{m}$ is also clustering and its cluster
functions are bounded by

$$
D \beta^{\lambda^{\prime} s} \prod\left(C \beta^{\lambda^{\prime}}\right)^{d_{\tau_{i}}} .
$$

Let us note that $E$ is also clustering with cluster functions

$$
\omega_{s}^{E}\left(\tau_{1}, \ldots, \tau_{s}\right)=\prod_{1}^{s} \omega_{1}^{E}\left(\tau_{i}\right)
$$

where

$$
\omega_{1}^{E}(\tau)=\left\{\begin{array}{ll}
1 & \tau=(\{t\},\{t\}) t \in \mathbb{Z}^{v} \\
0 & \text { otherwise }
\end{array} .\right.
$$

Moreover as $d_{(\{t\},\{t\})}=1$

$$
\left|\omega_{s}^{E}\left(\tau_{1}, \ldots, \tau_{s}\right)\right|<\left(\beta^{-\lambda}\right)_{1}^{\frac{1}{2} \sum_{1}^{s}|\tau|} \prod_{1}^{s}\left(\beta^{\lambda}\right)^{d_{\tau_{i}}}
$$

for any $\lambda$. If $\lambda<\lambda^{\prime}$ we get Lemma 3.1 from (3.3), (3.4), (3.8)-(3.10), (3.12).

Proof of Lemma 1.3. The operators

$$
B_{s, q}=\left(A_{22}^{(k)}\right)^{s} A_{21}^{(k)}\left(A_{11}^{(k)}\right)^{-(q+1)}
$$

$s, q \geqq 0$, are integers, and act from $L^{\leqq k}$ to $L^{>k}$. We shall look for $S^{(k)}$ as a series

$$
S^{(k)}=\sum x_{\alpha_{1}, \ldots, \alpha_{r}} B_{\alpha_{1}} A_{12}^{(k)} B_{\alpha_{2}} A_{12}^{(k)} \ldots A_{12}^{(k)} B_{\alpha_{r}}
$$


where the summation is over all sequences of pairs $\left(\alpha_{1}, \ldots, \alpha_{r}\right) r \geqq 1, \alpha_{i}=\left(s_{i}, q_{i}\right), s_{i}$ $\geqq 0, q_{i} \geqq 0, i=1, \ldots, r$, and $x_{\alpha_{1}, \ldots, \alpha_{2}}$ are real numbers to be found. Inserting (3.14) into the equation (1.21) one can get recurrent equations for $x_{\alpha_{1}, \alpha_{2}, \ldots, \alpha_{r}}$ For $r=1$

$$
\begin{gathered}
x_{(s, q)}=x_{(s-1, q-1)}, \quad \text { for } \quad s>0, \quad q>0, \\
x_{(0,0)}=1, \quad x_{(0, q)}=x_{(s, 0)}=0, \quad s, q>0 .
\end{gathered}
$$

For $r>1$

$$
x_{\alpha_{1}, \ldots, \alpha_{r}}=x_{\hat{\alpha}_{1}, \alpha_{2}, \ldots, \tilde{\alpha}_{r}}-\sum_{p=1}^{r-1} x_{\alpha_{1}, \ldots, \alpha_{p}} \cdot x_{\alpha_{p+1}, \ldots, \tilde{\alpha}_{r}},
$$

where for $\alpha=(s, q)$ we denote $\hat{\alpha}=(s-1, q), \tilde{\alpha}=(s, q-1)$. From (3.15) one can get immediately that

$$
x_{s, q}=\delta_{s, q} \quad s, q \geqq 0,
$$

where $\delta_{s, q}$ is the Kronecker symbol.

For any sequence $\left(\alpha_{1}, \ldots, \alpha_{r}\right), r>1, \alpha_{i}=\left(s_{i}, q_{i}\right)$ we introduce the sequence $\left(S_{i}, Q_{i}\right), i=1, \ldots, r$ of pairs $S_{i}=\left(s_{1}+s_{2}+\ldots+s_{i}\right), Q_{i}=\left(q_{1}+q_{2}+\ldots+q_{i}\right)$. Let us put

$$
K_{i}=Q_{i}-S_{i}-(i-1), \quad i=1,2, \ldots, r .
$$

We call the sequence $\left(\alpha_{1}, \ldots, \alpha_{r}\right)$ regular if

$$
K_{i} \geqq 0, \quad i=1,2, \ldots, r-1, \quad K_{r}=0 .
$$

If $\alpha_{1}, \ldots, \alpha_{r}$ is not regular then $\left(\hat{\alpha}_{1}, \alpha_{2}, \ldots, \tilde{\alpha}_{r}\right)$ is not regular also and for any $P$ one of the sequences $\left(\alpha_{1}, \ldots, \alpha_{p}\right)$ or $\left(\alpha_{p+1}, \ldots, \tilde{\alpha}_{r}\right)$ is not regular. Then (3.16) will be satisfied for nonregular sequences if we put

$$
x_{\alpha_{1}, \ldots, \alpha_{r}}=0
$$

for any nonregular sequence $\left(\alpha_{1}, \ldots, \alpha_{r}\right)$.

Then $x_{\alpha_{1}, \ldots, \alpha_{r}}$ for regular sequences are uniquely defined from (3.16-3.18). We shall consider further the solutions of (3.15), (3.16) just defined. Let us consider recurrent relations

$$
y_{\alpha_{1}, \ldots, \alpha_{r}}=y_{\hat{\alpha}_{1}, \ldots, \tilde{\alpha}_{r}}+\sum_{p=1}^{z-1} y_{\alpha_{1}, \ldots, \alpha_{p}} y_{\alpha_{p+1}, \ldots, \tilde{\alpha}_{r}},
$$

and consider solutions of these relations which are not equal to 0 for regular sequences. We have

Let us define

$$
\begin{gathered}
y_{\alpha_{1}, \ldots, \alpha_{r}} \geqq 0, \\
\left|x_{\alpha_{1}, \ldots, \alpha_{r}}\right| \leqq y_{\alpha_{1}, \ldots, \alpha_{r}} .
\end{gathered}
$$

$$
y_{S, r}=\sum y_{\alpha_{1}, \ldots, \alpha_{r}},
$$

where the summation is over all (regular) sequences with $S_{r}=S$ (and so $\left.Q_{r}=S-r-1\right)$. It follows from (3.19) that

$$
y_{S, r}=y_{S-1, \mathrm{r}}+\sum_{\substack{1 \leq p \leq r-1 \\ S_{1}+\bar{S}_{2}=S}} y_{S_{1}, p} y_{S_{2}, r-p}
$$


where

$$
y_{-1, r}=0, \quad r \geqq 1, \quad y_{S, 1}=1 .
$$

Then $y_{S, z}$ are uniquely defined by (3.22). (3.23). Let us consider the function $w=w(z, \varrho)$ of two complex variables $z, \zeta$. Let $w$ satisfy the following equation

$$
w=\zeta+z w+w^{2}, \quad w(0,0)=1 .
$$

Then $w$ is analytic for $z$ and $\zeta$ sufficiently small and can be expanded in a power series

$$
w(z, \zeta)=\sum_{S, r} w_{S, r} z^{S} \zeta r
$$

which is convergent for small $z, \zeta$.

It follows from (3.24) that $w_{s, r}$ satisfy (3.22), (3.23) and so

$$
w_{S, r}=y_{S, r}, \quad S \geqq 0, \quad r \geqq 1,
$$

and the series

$$
\sum_{r \leqq 1} \sum_{\left(\alpha_{1}, \ldots, \alpha_{r}\right)}\left|x_{\alpha_{1}, \ldots, \alpha_{r}}\right||z|^{S_{r}}|\zeta|^{r}
$$

is convergent for $z, \zeta$ sufficently small. We turn now to the investigation of the operators

$$
B_{\alpha_{1}, \ldots, \alpha_{r}}=B_{\alpha_{1}} A_{12}^{(k)} B_{\alpha_{2}} \ldots A_{12}^{(k)} B_{\alpha_{r}}
$$

for regular

Lemma 3.2. If $\left(\alpha_{1}, \ldots, \alpha_{r}\right)$ is regular then $B_{\alpha_{1}, \ldots, \alpha_{r}}$ is clustering and its cluster functions satisfy the following bounds

$$
\left|\omega_{s}\left(\tau_{1}, \ldots, \tau_{s}\right)\right|<(L \beta)^{2\left(S_{r}+2 r-1\right)} \prod\left(\beta^{\lambda}\right)^{d_{\tau_{i}}}
$$

where $L$ is an absolute constant.

Proof. As $\sum_{i=1}^{s} d_{\tau_{i}} \geqq \sum_{1}^{s}\left|\tau_{i}\right|-s$, we get

$$
\prod_{1}^{s} \beta^{d \tau_{i}} \leqq \prod_{1}^{s}\left(\beta^{\tilde{\lambda}}\right)^{d \tau_{i}} \beta^{(1-\tilde{\lambda})}\left[\sum_{1}^{s}\left|\tau_{i}\right|-s\right],
$$

where $0 \leqq \tilde{\lambda} \leqq 1$ and so the cluster functions of the operators $A_{\delta}^{(k)}, \delta=(12),(21),(22)$ have the following estimates

$$
\left|\omega_{s}^{A^{(k)}}\left(\tau_{1}, \ldots, \tau_{s}\right)\right|<M \beta^{(1-\lambda)(N+1)} \prod_{i=1}^{s}\left(\beta^{\lambda}\right)^{d_{\tau_{i}}} .
$$

Using Corollary 2.4 and (3.1) one can get that cluster functions of $B_{\alpha_{1}, \ldots, \alpha_{r}}$ satisfy the following bounds for small $\beta$

$\left|\omega_{s}\left(\tau_{1}, \ldots, \tau_{s}\right)\right|<\left(M \beta^{(1-\lambda)(N+1)}\right)^{S_{r}+2 r-1}\left[C_{N} \beta^{-N(\lambda+1)}\right]^{\left(Q_{r}+r\right)}\left(C \beta^{\lambda}\right)^{\left(S_{r}+Q_{r}+3 r-2\right) s} \prod_{i=1}^{s}\left(C \beta^{\lambda}\right)^{d_{\tau_{1}}}$. 
Using the fact that $Q_{r}=S_{r}+r-1$ for regular sequences and $\lambda<(N+1)^{-1}$, we can get (3.28) from (3.30). From (3.28) and (3.26) we get convergence of the series (3.14) for small $\beta$. The sum of (3.14) is the clustering operator and

$$
\left|\omega_{s}^{S^{(k)}}\left(\tau_{1}, \ldots, \tau_{s}\right)\right|<K \beta^{4} \prod_{i=1}^{s}\left(C \beta^{\lambda}\right)^{d_{\tau_{i}}}
$$

for some constant $K>0$.

Using (3.31) one can check (see [1]) that the sum of the series (3.14) has a sufficiently small norm and coincides with the unique solution of (1.21) having a small norm. Lemma 1.3 is proved.

Proof of Lemma 1.4. We have

$$
\operatorname{Im} \tilde{V}^{(k)}=\tilde{V}^{(k)} L^{k} \subset \mathscr{H}_{k} .
$$

We shall prove $\operatorname{Im} \tilde{V}^{(k)}=\mathscr{H}_{k}$.

We shall show first that

$$
\begin{aligned}
E-P_{\mathscr{L}^{(k-1)}}= & {\left[E-\left(S^{(k-1)}\right)^{*}\right]\left[E_{L^{>(k-1)}}+S^{(k-1)}\left(S^{(k-1)}\right)^{*}\right] } \\
& \cdot\left[P_{L^{>(k-1)}}-\left(S^{(k-1)}\right)^{*} P_{L^{\leq k-1}}\right],
\end{aligned}
$$

where

$$
\left[E_{L^{>(k-1)}}+S^{(k-1)}\left(S^{(k-1)}\right)^{*}\right]^{-1} \text { acts in } L^{>(k-1)} .
$$

In fact for any $f \in l_{2}\left(\mathbf{C}_{\mathbb{Z}^{v}}\right)$

$$
\begin{gathered}
P_{\mathscr{L}^{(k-1)}} f=\varphi+S^{(k-1)} \varphi, \quad \varphi \in L^{\leqq(k-1)} \\
\left(E-P_{\left.\mathscr{L}^{(k-1)}\right)}\right) f=\psi+\left(S^{(k-1)}\right)^{*} \psi, \quad \psi \in L^{>(k-1)} .
\end{gathered}
$$

As

$$
S^{(k-1)} \varphi \in L^{>(k-1)}, \quad\left(S^{(k-1)}\right)^{*} \psi \in L^{\leqq(k-1)},
$$

one has that

$$
\begin{aligned}
& P_{L^{\leq k(k-1)}} f=\varphi-\left(S^{(k-1)}\right)^{*} \psi \\
& P_{L^{>(k-1)}} f=\psi+\left(S^{(k-1)}\right) \varphi
\end{aligned}
$$

It follows that

$$
\psi+S^{(k-1)}\left(S^{(k-1)}\right)^{*} \psi=P_{L^{>(k-1)}} f-S^{(k-1)} P_{L^{\Xi(k-1)}} f,
$$

and

$$
\psi=\left[E_{L^{>(k-1)}}+S^{(k-1)}\left(S^{(k-1)}\right)^{*}\right]^{-1}\left(P_{L^{>(k-1)}}-S^{(k-1)} P_{\left.L^{\leq k-1}\right)}\right) f .
$$

Finally, (3.32) follows from (3.33). 
Lemma 3.3. The operator $E_{L^{>(k-1)}}+S^{(k-1)}\left(S^{(k-1)}\right)^{*}$ is invertible in $L^{>(k-1)}$ and its inverse is equal to

$$
\left[E_{L^{>(k-1)}}+S^{(k-1)}\left(S^{(k-1)}\right)^{*}\right]^{-1}=E_{L^{>(k-1)}}+G^{(k-1)},
$$

where $G^{(k-1)}$ is the clustering operator with clustering parameter $\beta^{\lambda}$. Moreover the norm $\left\|G^{(k-1)}\right\|$ is sufficiently small for $\beta$ sufficiently small.

The proof follows from the expansion

$$
\left[E_{L^{>(k-1)}}+S^{(k-1)}\left(S^{(k-1) *}\right]^{-1}=E_{L^{>(k-1)}}+\sum(-1)^{n}\left[S^{(k-1)}\left(S^{(k-1) *}\right]^{n}\right.\right.
$$

by Corollary 2.4 and (3.31). The estimate of the norm can be obtained by calculations similar to those in [1]. Let us note that it follows from (3.32) that for $f \in L^{>(k-1)}\left(E-P_{\mathscr{L}^{(k-1)}}\right) f=\left[E-\left(S^{(k-1)}\right)^{*}\right]\left(f+G^{(k-1)} f\right)$, and so

where

$$
\tilde{V}^{(k)} f=f+\tilde{\Gamma}^{(k)} f,
$$

$$
\begin{aligned}
\tilde{\Gamma}^{(k)}= & -\left(S^{(k-1)}\right)^{*}-\left(S^{(k-1)}\right)^{*} S^{(k)}+S^{(k)}+G^{(k-1)} \\
& -\left(S^{(k-1)}\right)^{*} G^{(k-1)}+G^{(k-1)} S^{(k)}-\left(S^{(k-1)}\right)^{*} G^{(k-1)} S^{(k)} .
\end{aligned}
$$

Again using Lemma 3.3, Corollary 2.4 and the estimate (3.31), one can find that $\tilde{\Gamma}^{(k)}$ is clustering with clustering parameter $\beta^{\tilde{\lambda}}$ and sufficiently small norm. So we proved that property 3) of $\tilde{V}^{(k)}$.

We shall prove property 1) of $\tilde{V}^{(k)}$. If $\tilde{V}^{(k)} L^{k} \neq \mathscr{H}_{k}$, then there exists $\Phi \in \mathscr{H}_{k}$ which is orthogonal to all vectors $f+S^{(k)} f \in \mathscr{L}^{(k)}$, where $f \in L^{k}$. Without restricting the generality one can assume that

$$
\|\Phi\|=1
$$

As $\Phi \in \mathscr{L}^{(k)}$, one can write

$$
\Phi=\varphi+S^{(k)} \varphi=f+\varphi_{1}+S^{(k)}\left(f+\varphi_{1}\right),
$$

where

$$
\varphi=\varphi_{1}+f \in L^{\leqq k}, \quad \varphi_{1} \in L^{\leqq(k-1)}, \quad f \in L^{k} .
$$

We have

$$
1=\|\Phi\|^{2}=\|\varphi\|^{2}+\left\|S^{(k)} \varphi\right\|^{2},
$$

and then

$$
\|\varphi\|<1
$$

Moreover from the equality

$$
\|\varphi\|^{2}=\left\|\varphi_{1}\right\|^{2}+\|f\|^{2},
$$

one can find that

$$
\left\|\varphi_{1}\right\| \leqq\|\varphi\|
$$

We have also

$$
0=\left(f+S^{(k)} f, \Phi\right)=\left(\varphi_{1}+S^{(k)} \varphi_{1}, \quad f+S^{(k)} f\right)+\left\|f+S^{(k)} f\right\|^{2} .
$$


As

$$
\left|\left(\varphi_{1}+S^{(k)} \varphi_{1}, \quad f+S^{(k)} f\right)\right|=\left|\left(S^{(k)} \varphi_{1}, \quad S^{(k)} f\right)\right| \leqq\left\|S^{(k)}\right\|^{2}\left\|\varphi_{1}\right\|\|f\|,
$$

and

$$
\left\|f+S^{(k)} f\right\|^{2}=\|f\|^{2}+\left\|S^{(k)} f\right\|^{2},
$$

one can find from $(3.37),(3.36 \mathrm{~b})$ that

$$
\|f\| \leqq \frac{\left\|S^{(k)}\right\|^{2}\|\varphi\|}{1-\left\|S^{(k)}\right\|^{2}} .
$$

Acting on both sides of (3.36) with the operator $E-P_{\mathscr{L}^{(k-1)}}$, one can get

$$
\Phi=\left(E-P_{\left.\mathscr{L}^{(k-1)}\right)}\right)\left(\varphi_{1}+S^{(k)} \varphi_{1}\right)+\tilde{V}^{(k)} f .
$$

Let us note that

$$
\begin{gathered}
\left(E-P_{\mathscr{L}^{(k-1)}}\right)\left(\varphi_{1}+S^{(k)} \varphi_{1}\right)=\left(E-\left(S^{(k-1)}\right)^{*}\right) \\
\left(E_{L^{>(k-1)}}+G^{(k-1)}\right)\left[-S^{(k-1)} \varphi_{1}+S^{(k)} \varphi_{1}\right] .
\end{gathered}
$$

From here and from (3.36a), (3.36b) it follows that

$$
\left\|\left(E-P_{\mathscr{L}^{(k-1)}}\right)\left(\varphi_{1}+S^{(k)} \varphi_{1}\right)\right\|<K_{1} \varepsilon_{1}(\beta),
$$

where $K_{1}$ is the absolute constant, $\varepsilon_{1}(\beta) \rightarrow 0$ if $\beta \rightarrow 0$.

From (3.38) one can find that

$$
\left\|\tilde{V}^{(k)} f\right\| \leqq K_{2} \varepsilon_{2}(\beta),
$$

where $K_{2}$ is the absolute constant, $\varepsilon_{2}(\beta) \rightarrow 0$ if $\beta \rightarrow 0$. Estimates (3.39) and (3.40) for $\beta$ sufficiently small, contradict (3.35a).

So we proved that

$$
\tilde{V}^{(k)} L^{k}=\mathscr{H}_{k}
$$

As $\tilde{V}^{(k)} f=f+\tilde{\Gamma}^{(k)} f$ for $f \in L^{k}$, where $\tilde{\Gamma}^{(k)}$ has small norm, $\tilde{V}^{(k)} f \neq 0$ for all $f \in L^{k}, f \neq 0$. Then $\operatorname{Ker} \widetilde{V}^{(k)}=l_{2} \ominus L^{k}$. Lemma 1.4 is proved.

Lemma 1.5 follows from the fact that $D_{k}=\left(\tilde{V}^{(k)}\right)^{*} \tilde{V}^{(k)}$ is the positive operator $\operatorname{Im} D_{k}=L^{k}, \operatorname{Ker} D_{k}=l_{2} \ominus L^{k}$, and moreover

$$
\begin{aligned}
\left(\tilde{V}^{(k)}\right)^{*} \tilde{V}^{(k)} & =\left(P_{L^{k}}+\left(\tilde{\Gamma}^{(k)}\right)^{*}\right)\left(P_{L^{k}}+\tilde{\Gamma}^{(k)}\right) \\
& =P_{L^{k}}+P_{L^{k}} \tilde{\Gamma}^{(k)}+\left(\Gamma^{(k)}\right)^{*} P_{L^{k}}+\left(\tilde{\Gamma}^{(k)}\right)^{*} \tilde{\Gamma}^{(k)}=P_{L^{k}}+\hat{\Gamma}^{(k)}
\end{aligned}
$$

where

$$
\hat{\Gamma}^{(k)}: L^{k} \rightarrow L^{k}
$$

is a selfadjoint clustering operator with small norm. It follows that

$$
\left[\left(\tilde{V}^{(k)}\right)^{*} \tilde{V}^{(k)}\right]^{-1 / 2}=P_{L^{k}}+\sum_{s \geqq 1} \alpha_{s}\left(\hat{\Gamma}^{(k)}\right)^{s}
$$

where $\alpha_{s}$ are the coefficients of the expansion

$$
(1+x)^{-1 / 2}=1+\sum \alpha_{s} x^{s}, \quad|x|<1
$$


After applying Lemma 1.2I to each term of (3.42), the proof of Lemma 1.5 is completed. Proof. of Lemma 1.6 is quite evident.

\section{References}

1. Malyshev, V.A., Minlos, R. A.: Invariant subspaces of clustering operators. I., J. Stat. Phys. 21, 231242 (1979)

2. Malyshev, V.A.: Uniform cluster estimates for lattice models. Commun. Math. Phys. 64, 131-157 (1979)

3. Malyshev, V.A.: Uspehi Mat. Nauk 45, 3-53 (1980) (in Russian)

Communicated by Ya. G. Sinai

Received March 15, 1981

Note added in proof. I. Kashapov and the first author proved recently for the similar class of clustering operators that zero is not an eigenvalue of clustering operator. 